\title{
Divulgación
}

\section{Periimplantitis: fisiopatología y diagnóstico mediante pruebas bioquímicas.}

Periimplantitis: pathophysiology and di-

agnosis by biochemical tests.

Fecha de Recepción

27 de mayo de 201 I
Periimplantite: fisiopatologia e diagnóstico por testes bioquímicos.

Aceptado para su publicación

30 de junio de 2011
Auxiliar Docente I ra. Fisiología Humana. Facultad de Odontología. Universidad Nacional del Nordeste.

Miguel Jorge Acuña Auxiliar Docente I ra. Física - Química Biológica y Periodoncia. Facultad de Odontología. Universidad

Nacional del Nordeste.

Rolando Pablo Juárez Profesor Titular. Fisiología Humana. Facultad de Odontología. Universidad Nacional del Nordeste.

Daniel Antonio Pira Profesor Adjunto. Clínica de Prótesis I Curso. Facultad de Odontología. Universidad Nacional del

Nordeste.

\section{Resumen}

El objetivo de este trabajo fue realizar una revisión de la bibliografía internacional sobre la enfermedad periimplantaria, destacando los aspectos fisiopatológicos y su diagnóstico.

Los procesos periimplantarios son infecciones por biofilms bacterianos de implantes osteointegrados, que pueden resultar en la pérdida implantaria y la disminución de los niveles óseos que resulta en una grave complicación en nuestra clínica pues dificulta aún más el tratamiento de los pacientes edéntulos.

El conocimiento de la fisiopatología y biología molecular es clave en la detección de patología periimplantaria, permitiendo de esta manera una acción terapéutica precoz para evitar la pérdida de implantes dentales y la pérdida ósea asociada.

\section{Palabras Claves}

Periimplantitis, biología molecular, diagnóstico.

\section{Summary}

The objective of this work was to conduct a review of international literature on the disease periimplantaria, emphasizing the aspects pathophysiological and its diagnosis.

The periimplantarios processes are infections by bacterial biofilms of implants osseointegrated, which can result in the loss implantaria and the decrease of bone levels resulting in a serious complication in our clinic because it makes it difficult to even more treatment of the edentulous patients. 
Knowledge of the pathophysiology and molecular biology is key in the detection of pathology periimplantaria, allowing an early therapeutic action to avoid the loss of dental implants and associated bone loss.

\section{Keywords}

Periimplantitis, molecular biology, diagnosis.

\section{Resumo}

O objetivo deste trabalho foi de realizar uma revisão da literatura internacional sobre a doença periimplantaria, enfatizando os aspectos fisiopatológico e seu diagnóstico.

Os processos periimplantarios são infecções causadas por bactérias biofilmes de implantes osseointegrated, que pode resultar em implantaria a perda e a diminuição dos níveis de osso, resultando em uma complicação grave em nossa clínica, pois ele torna difícil a um tratamento ainda mais dos pacientes desdentados.

Conhecimento da fisiopatologia e biologia molecular é a chave para a detecção da patologia periimplantaria, permitindo uma ação terapêutica cedo evitar a perda de implantes dentários and perda óssea associado.

\section{Palavras chave}

Periimplantitis, biologia molecular, diagnóstico.

\section{Introducción}

Los implantes dentales de titanio se están utilizando desde hace muchos años en la rehabilitación de pacientes, tanto total como parcialmente edéntulos, con una efectiva tasa de éxito a largo plazo, comprendida entre un $90 \%$ y un $98 \% \%^{1-3}$.

A pesar de los resultados satisfactorios, los tejidos que soportan los implantes osteointegrados son susceptibles de patologías que pueden llevar a la pérdida del implante 4 .

La periimplantitis fue definida como un proceso inflamatorio que afecta a los tejidos que rodean al implante osteointegrado en función, con la consecuente pérdida de hueso de soporte y de la osteointegración $n^{5}$.

Es la placa bacteriana, en forma de biofilm, la que afecta a los tejidos peri-implantarios produciendo una lesión inflamatoria de mayor o menor profundidad.
Cuando la reacción inflamatoria queda confinada en el tejido blando que rodea al implante se denomina mucositis, respuesta reversible si se elimina la causa. Pero, cuando la inflamación progresa apicalmente afectando también al tejido duro estamos ante una peri-implantitis, fenómeno no reversible, con pérdida de hueso de soporte del implante ${ }^{6}$.

El objetivo de este trabajo es hacer tomar conciencia al odontólogo de la importancia de la detección precoz de la patología periimplantaria y actuar tempranamente para evitar en lo posible la pérdida implantaria y ósea.

\section{Fisiopatología}

Dentro del proceso fisiopatológico de la periimplantitis es importante reconocer los factores etiológicos, microorganismos presentes y los mediadores químicos involucrados en el mismo.

Se ha sugerido que estos fracasos tardíos son por lo general el resultado de una carga excesiva y/o infección. Respecto de los fracasos tardíos por infección, en el Workshop Europeo de Periodontología conducido por la Federación Europea de Periodontología se acuñaron denominaciones para dos patologías inflamatorias periimplantarias bien diferenciadas: mucositis periimplantaria y periimplantitis ${ }^{7}$.

La porción coronal, el transmucoso o el emergente de los implantes osteointegrados sirve como nicho para la colonización bacteriana de biopeliculas. Como ocurre en un diente natural la mucosa periimplantaria se encuentra en intima relación con el mismo y esto lleva a que en la superficies de los mismos puedan desarrollarse colonias bacterianas patógenas.

Esta colonización conlleva reacciones inflamatorias en los tejidos periimplantarios que presentan analogías con el proceso de la periodontitis.

En pacientes parcialmente desdentados la microbiota bacteriana es muy similar a la que se encuentra alrededor de los implantes dentarios; observándose en la zona subgingival alrededor de los implantes una mayor cantidad de espiroquetas y bacilos móviles en comparación con dientes residuales del mismo maxilar ${ }^{8,9}$.

Una historia de periodontitis y la presencia de agentes patógenos periodontales son factores que pueden influir en la condición de los tejidos 
blandos periimplantarios en pacientes parcialmente desdentados ${ }^{10}$.

Mediante microscopía de contraste de fase y sondeos de ADN, se determinó que los perfiles microbiológicos fueron similares alrededor de los dientes y los implantes dentales en profundidades de bolsa similares ${ }^{11,12}$.

Un mes después de la colocación de los implantes ya se detectaron agentes patógenos periodontales alrededor de los mismos en pacientes parcialmente desdentados ${ }^{13}$.

En pacientes desdentados rehabilitados con implantes dentarios que sufrieron de periimplantitis se han visto asociados microorganismos como Actinobacillus actinomycetemcomitans y Porphyromonas gingivalis ${ }^{14}$. Además, se han observado otras especies bacterianas en relación con la perimplantits como por ejemplo Bacteroides forsythus, Fusobacteria nucleatum, Campylobacter, Peptostreptococcus micros y Fusobacterium intermedia ${ }^{15}$.

Microorganismos que suelen estar menos asociadosconlaperiodontitis, talescomoStaphylococcus spp y Candida spp, también se han encontrado en infecciones de peri-implante ${ }^{16,17}$.

El modelo actual de etiopatogénesis de la enfermedad periodontal establece que bacterias periodontopáticas como Porphyromonas gingivalis, Bacteroides forsythus y Actinobacillus actinomycetemcomitans son agentes causales primarios. Su introducción como infección exógena junto con una microbiota patogénica dispara una cascada de respuestas en el hospedero. Una respuesta inmediata es el reclutamiento y migración de leucocitos PMNs al sitio de la infección periodontal. Si estas células inflamatorias son capaces de contener y eliminar los patógenos causales y sus productos (como el LPS o lipopolisacárido de las bacterias Gram negativas) vía fagocitosis y mecanismos de eliminación intracelular, la enfermedad se limita a gingivitis. Si estos mecanismos fallan, y los patógenos o sus productos penetran los tejidos del hospedero, la enfermedad se convierte en periodontitis. El eje monocitario-linfocitario del hospedero se estimula y localmente libera mediadores inflamatorios, como los metabolitos del ácido araquidónico (PGE2, leucotrienos y lipoxinas) y citoquinas proinflamatorias (IL-Iß, IL-6 y TNF- $\alpha$ ). Estos mediadores inflamatorios a largo plazo causan destrucción tisular local, y clínicamente se establece con la formación de la bolsa periodontal y la pérdida de hueso alveolar. Además, las condiciones ambientales locales secundarias de la inflamación y los eventos degradativos (baja tensión del oxígeno y disponibilidad de hierro) soportan la microbiota patogénica y perpetúan el ciclo patogénico ${ }^{18}$.

\section{Diagnóstico de la periimplantitis}

Múltiples métodos de diagnóstico son conocidos y determinan el grado de destrucción que presentan los tejidos periodontales a la hora de un examen clínico, pero no muchos se ocupan en determinar la actividad o no actividad molecular y/o enzimática.

El diagnóstico de periimplantitis ha sido claramente establecido en los Proceedings of the 3rd. European Workshop on Periodontology ${ }^{(7)}$ mediante la constatación de una serie de signos irrefutables: a) debe existir evidencia radiológica de destrucción vertical de la cresta ósea; b) hay sangrado después del sondeo suave, pudiendo existir supuración de la bolsa; c) los tejidos blandos marginales pueden estar inflamados, tumefactos y enrojecidos.

La periimplantitis en la mayoría de los casos es diagnosticada cuando ya existen evidencias clínicas y cuando ya el proceso involucra perdida de incersion alrededor del implante, pues el proceso fisiopatologico empieza sin evidencia clínica. Radiológicamente, se puede detectar el problema cuando ya se ha perdido el $30 \%$ de la masa ósea, por lo que no es un método adecuado para hacer el diagnóstico precoz de la periimplantitis ${ }^{19}$. Cuando la pérdida ósea se debe a causas infecciosas, se detecta la presencia de bacterias gramnegativos, espiroquetas y microorganismos móviles, supuración, aumento de profundidad y sangrado al sondaje, índice gingival y de placa aumentados, dolor a la masticación y la presencia de tejido de granulación periimplantario ${ }^{20}$.

Mediante el análisis del fluido del surco periimplantario, pueden detectarse algunos cambios precoces que demuestran la existencia de reabsorción ósea, como es el aumento del nivel de condroitín sulfato, igual que sucede en las parodontopatías crónicas no tratadas o en pacientes bajo tratamiento ortodóncico. La elastasa, la $\beta$-glucuronidasa, la aminotransferasa y la prostaglandina E2 también están elevadas ${ }^{21}$.

A nivel molecular para la identificación de los 
microorganismos colonizadores de la bolsa pueden usarse sondas de DNA capaces de identificar secuencias de nucleótidos específicos de determinadas especies ${ }^{22}$.

Además estudios enzimaticos como el BANA o determinación de aumento de fosfatasa alcalina podrían ser útiles como marcadores bioquímicos de destrucción tisular y perdida ósea cercana al implante ${ }^{23}$.

Existen pruebas diagnósticas para detectar determinados patógenos. El cultivo microbiológico es una de las más frecuentes, pudiéndose realizar mediante una muestra del exudado periimplantario obtenida utilizando puntas de papel estéril o tomando la muestra con micro pipetas calibradas. Ademas se han desarrollado sondas de ADN (ácido desoxirribonucleico) para identificar las secuencias de nucleótidos específicos de determinadas especies ${ }^{24}$. Es aconsejable realizar un antibiograma para conocer la sensibilidad antibiótica de la flora microbiana subgingival periimplantaria.

También se ha admitido como pruebas válidas para la detección precoz de la patología inflamatoria periimplantaria el registro de la temperatura gingival y el registro del volumen del fluido periimplantario ${ }^{25}$.

\section{Conclusiones}

Se podría considerar que la enfermedad periimplantaria en los últimos años se ha ubicado en un lugar importante a tener en cuenta por el odontólogo a la hora de pronosticar una rehabilitación con la colocación de implantes.

Se reconocen diferentes factores de riesgo de contraer periimplantitis, la falta de higiene oral, pacientes con antecedentes de periodontitis y el hábito de fumar se encuentran en mayor medida. Además se puede mencionar factores genéticos, diabetes, consumo de alcohol y propiedades de la superficie del implante.

Después de toda la bibliografía revisada, podemos aseverar que la periimplantitis no se producirá si la identificación y tratamiento del proceso se realiza precozmente; en ese diagnóstico precoz las pruebas bioquímicas resultan de gran importancia.

\section{Bibliografía}

I. Adell R, Eriksson B, Lekholm U, Branemark PI, Jemt T. A long term follow-up study of osseointegrated implants in the treatment of totally edentulous jaws. Int J Oral Maxillofac Implants 1990; 5 (4): 347 - 59.

2. Albrektsson T, Isidor F. Consensus report of session IV. In: Lang NP y Karring T (eds.). Proceedings of the Ist European Workshop on Periodontology. Londres: Quintessence; 1994.

3. Alonso Alvarez B; Legido Arce B. ¿Qué conocimientos tenemos actualmente sobre la periimplantitis, sus causas, su diagnóstico y su tratamiento? Cient Dent 2010; 7 (3): 179 - 182.

4. Jemt $T$, Linden B, Lekholm U. Failures and complications in 127consecutively placed fixed postheses supported by Bränemark implants: from the treatment to firs annual check-up. Int J Oral Maxillofac Implants 1992; 7 (I): 40 - 44.

5. Lekholm U, van Steenberghe D, Herrmann I, Bolender C, Folmer T, Gunne J. Osseointegrated implants in the treatment of partially edentulous jaws. A prospective 5 year multicenter study. Int J Oral Maxillofac Implants 1994; 9 (6): 62I - 35.

6. Fardal O, Johannessen AC, Olsen I. Severe, rapidly progressing peri-implantitis. J Clin Periodontol 1999; 26 (5): $313-7$.

7. Lang N, Karring T, Lindhe J. Proceedings of the 3rd. European Workshop on Periodontology Implant Dentistry 1999; Quintessence Publising Co.

8. Leonhardt A, Adolfsson B, Lekholm U, et al. A longitudinal microbiological study on osseointegrated titanium implants in partially edentulous patients. Clin Oral Implants Res1993; 4: II3 $-20$.

9. Mombelli A, Marxer M, Gaberthuel T, et al. The microbiota of osseointegrated implants inpatients with a history of periodontal disease. J Clin Periodontol 1995; 22: 124 - 30.

10. Quirynen M, Listgarten M. The distribution of bacterial morphotypes around natural teethand titanium implants ad modum $\mathrm{Bra}^{\circ}$ nemark. Clin Oral Implants Res 1990; I: 8 - 13.

II. Papaioannou W, Quirynen M, Van Steenberghe $D$. The influence of periodontitis on the subgingival flora around implants in partially edentulous patients. Clin Oral Implants Res 1996; 7: 405-9.

12. Van Winkelhoff AJ, Goene RJ, Folmer T. Early colonization of dental implants by putative periodontal pathogens in partially edentulous patients. Clin Oral Implants Res 2000; II: 51 I - 20.

13. Leonhardt A, Adolfsson B, Lekholm U, et al. A longitudinal microbiological study on osseointe- 
grated titanium implants in partially edentulous patients. Clin Oral Implants Res 1993; 4: II3 - 20.

14. Mombelli A, van Oosten MA, Schurch E Jr, et al. The microbiota associated with successful or failing os seointegrated titanium implants. Oral Microbiol Immunol 1987; 2: I45 - 5 I.

15. Tanner A, Maiden MF, Lee K, et al. Dental implant infections. Clin Infect Dis 1997; 25 (Suppl 2): S 213- 7.

16. Slots J, Rams TE. New views on periodontal microbiota in special patient categories. J Clin Periodontol I99|; |8: 4II - 20.

17. Leonhardt A, Renvert S, Dahle'n G. Microbial findings at failing implants. Clin Oral Implants Res 1999; 10: 339 - 45.

18. Arce RM. Terapia periodontal del futuro. Colombia Médica 2004; 35 (3), (Supl I): 40 - 47.

19. Alonso Alvarez B; Legido Arce B. ¿Qué conocimientos tenemos actualmente sobre la periimplantitis, sus causas, su diagnóstico y su tratamiento? Cient Dent 2010; 7 (3): 179 - 182.

20. Jemt $T$, Linden $B$, Lekholm U. Failures and complications in 127consecutively placed fixed postheses supported by Bränemark implants : from the treatment to firs annual check-up. Int J Oral Maxillofac Implants 1992; 7: 40 - 44.

21. Lekholm U, van Steenberghe D, Herrmann I, Bolender C, Folmer T, Gunne J. Osseointegrated implants in the treatment of partially edentulous jaws. A prospective 5 year multicenter study. Int J Oral Maxillofac Implants 1994; 9: 621 - 35.

22. Fardal O, Johannessen AC, Olsen I. Severe, rapidly progressing peri-implantitis. J Clin Periodontol 1999; 26 (5): 313 - 7.

23. Sánchez M, Gay C. Periimplantitis. Med Oral Patol Oral Cir Bucal. 2004; 9: 63 - 74.

24. Waddington RJ, Embery G. Proteoglycans and orthodontic tooth movement. J Orthod 200I; 28 (4): 28 I - 90.

25. Van Winkelhoff AJ. Consensus on peri-implant infections. Oct.; 117 (I0): 519 - 23. 2010. 